\title{
AMIGM: ANIMAL MIGRATION INSPIRED GROUP MOBILITY MODEL FOR MOBILE AD HOC NETWORKS
}

\author{
JYOTSNA VERMA AND NISHTHA KESSWANI*
}

\begin{abstract}
The most widespread notion of mobility model is the representation of mobile nodes movement pattern in the wireless ad hoc networks which has a significant impact on the performance of the network protocols. In this paper, we have proposed an Animal Migration Inspired Group Mobility (AMIGM) model for mobile ad hoc networks based on the migration behavior of animals like, insects, flock of birds, schools of fishes, reptiles, amphibians, etc. The propound model tries to overcome the limitations of the existing mobility models, such as temporal dependencies, spatial dependencies, geographical restrictions and migration of nodes between the group of nodes so that it can realistically model the real world application scenarios. The proposed AMIGM model is based on Animal Migration Optimization algorithm, in which each group of nodes has two phases namely, Migration phase and Population updating phase. In the first phase, the model simulates the movement of nodes in the group from one position to another by obeying the swarming laws. In the second phase, the model simulates joining and leaving of the nodes in the group during migration. The protocol dependent and independent performance metrics of the proposed model are compared with Random Waypoint Mobility model and Reference Point Group Mobility model through ns-2 simulator.
\end{abstract}

Key words: Animal migration optimization, Mobility model, Ad hoc Networks, RWP, RPGM.

AMS subject classifications. 68M10

1. Introduction. The evolution of Mobile Ad hoc Networks (MANETs) over the years is potentially one of the major advances in the history of wireless networking. MANET is basically an autonomous system of mobile nodes connected by wireless links without any fixed infrastructure. It is characterized by multi-hop wireless links, shared radio channel, distributed routing, packet switched network, quick and low cost of deployment, selforganization and maintenance [1]. These characteristics of MANETs led to a diverse range of applications, such as emergency and rescue operations, disaster recovery, transportation systems, military applications, security operations, environmental monitoring, conferences and smart homes. Nodes in MANETs are highly mobile and can move in any direction with any speed which leads to frequent path breaks and affects the performance of wireless ad hoc networks, such as connectivity of the nodes, communication traffic, network protocols, etc., $[2,3]$. Mobility model is a critical and important parameter for a system that specifies the movement pattern of mobile nodes. Thus, it is significant to emulate the real world applications. Various individual as well as group mobility models exist in the literature that tries to realistically model the different movement patterns of the nodes, such as Random Waypoint (RWP) model [4] and Reference Point Group Mobility (RPGM) model [5] etc. The performance of wireless ad hoc networks varies considerably with different mobility models and their parameters during simulation. Thus, an efficient mobility model is required that can model the realistic scenarios.

In view of the necessity of developing efficient, realistic mobility model, the paper proposes an AMIGM model based on swarming rules and Animal Migration Optimization (AMO) algorithm [6] which is a new heuristic optimization method based on the animal swarm migration behavior. In the proposed model, each group of nodes in the network has two phases: Migration phase and Population updating phase. In the first phase, the model simulates the movement of nodes in the group from one position to another by obeying the swarming rules such as cohesion, homing, dispersion, collision avoidance, etc., to direct their movement in the network. In the second phase, the model simulates joining and leaving of the nodes in the group during migration. Thus, our model is capable of modeling the migration of nodes between the groups based on AMO algorithm and can avoid inter-intra group collision, environmental obstacles which mathematically model the movement pattern of the nodes in mobile ad hoc networks.

1.1. Motivation. Mobility is the salient feature of MANETs. So, researchers have designed many mobility models over the past years to mimic the realistic scenarios, but fail to recreate the realistic applications for the MANETs, such as migration of nodes from one group to another group, collision avoidance among the nodes,

*Department of Computer Science, Central University of Rajasthan, Ajmer, Rajasthan, India. (jyotsna.verma.mca@gmail.com). This research is supported by the NFO Fellowship under the University Grants Commission 
avoidance of geographical restrictions etc. Hence, to realistically represent the movement pattern of the nodes, researchers are eventually approaching towards the bio inspired techniques where the solutions of the problems are inspired from swarm behavior of natural systems, like insects, flock of birds, schools of fishes, reptiles, amphibians, etc. [7]. Characteristics of bio inspired approaches, like cooperative movement, no permanent membership, speed, adaptation, scalability, fault tolerance, modularity, parallelism and autonomy etc., suit the application of ad hoc wireless networks.

There are many mobility models $[8,9,10,11]$ in the literature that use nature inspired techniques to model the realistic scenarios and till date, to the best of our knowledge, there is no generalized mobility model for all the application scenarios. The approach of our proposed mobility model is based on the animal migration behavior ecology which can be found in all animal groups, like fish, reptiles, amphibians, insects, etc. Generally, the cause of animal migration is due to climate change, food, seasons, etc. So, the main attribute of the work is to model the migration behavior of the animals from one group to another group to achieve realistic mobility model.

1.2. Contributions. The principal contributions of this paper are as follows:

1. We have proposed an Animal Migration Inspired Group Mobility (AMIGM) model for MANETs based on the AMO approach and some swarming laws for the governing of the group mobility. It is capable of modeling the migration behavior of the nodes from one group to another group by replacing the worst fitness nodes of one group with the best fitness nodes from the other group of nodes.

2. Mathematical models of the group formation and migrating procedures are presented.

3. We have compared AMIGM model with the two prevailing existing mobility models: RWP [4] and RPGM [5].

4. Finally, the effect of mobility models on network performance with respect to the mobility under Dynamic Source Routing (DSR) protocol is evaluated.

5. The simulation results for the proposed AMIGM model shows the least average energy consumption with highest average link duration and average degree of spatial dependence when compared with existing models.

The rest of the paper is structured as follows: Section 2 highlights the related works. Section 3 presents the proposed Animal Migration Inspired Group Mobility (AMIGM) model. Section 4 presents the algorithm for the proposed AMIGM model and Section 5 analyses the time complexity of the proposed AMIGM algorithm. Section 6 presents the simulation environment and results of the protocol dependent and independent performance of mobility models in MANETs under the DSR routing protocol. Section 7 presents the movement pattern of the nodes correspond to the different mobility models and Section 8 summarizes the paper.

2. Related Work. The emergence of the phenomenon commonly known as mobility model represents the movement pattern of the nodes and how their velocity, acceleration, and location changes over time. Considering the mobility characteristics of the nodes in MANETs, formulation of the realistic mobility model is very important as an unrealistic model gives unrealistic results which will ultimately affect the performance of the mobility models. There are many mobility models $[3,4,5,12,13]$ in the literature that try to create the realistic application scenarios.

Broadly, mobility models are classified into three categories: Trace based mobility models, Stochastic/Individual mobility models and Synthetic/Group mobility models. Trace based mobility models are based on real traces, but due to the limited public repository of traces, it is difficult to model the nodes in real world scenarios. Stochastic mobility models are idealistic models where nodes move independently within the network without imposing the constraints like obstacles, pathways, etc. Random walk mobility model [14], Random waypoint mobility model [4], Random direction mobility model [15] etc., are some of the stochastic/individual/random based mobility models. The Random walk mobility model also called as Brownian motion is the most popular and simple mobility model which is first described mathematically by Einstein in (1926). In this, mobile nodes independently move from one location to another location with randomly chosen direction and speed from predefined ranges $[0,2 \pi]$ and [minspeed, maxspeed] respectively. Another popular mobility model is the Random waypoint mobility model in which nodes independently move around the simulation area, including pause time between randomly chosen direction and speed. The Node begins its movement by staying at one location for specific pause time say for $\mathrm{t}$ second and then it moves again to another location within the simulation bound- 
ary in an inconstantly chosen direction and speed from the ranges between $[0,2 \pi]$ and [minspeed,maxspeed] respectively. The Random direction mobility model, on the other hand, chooses a random direction and move along that direction until it reaches the simulation boundary. On reaching the simulation boundary, it again chooses a direction and repeats this process until the simulation time is reached. Random movement of nodes sometimes creates the interference in the network. Various mobility models found in the literature deals with the interference in the network. In [16] authors map the variation of distance caused by the nodes mobility to the variation in channel gain for describing the distinctive nature of the interference statistics of random networks. For handling complex mobility and predicting time varying interference authors in [17] proposes a general mobility model for a finite number of nodes using a general-order linear model in MANETs.

The individual mobility models are simple to implement, but they do not represent the group mobility behavior of the nodes in the network. This induces the development of stochastic/group mobility models. Group mobility models are the mathematical models which are spatially dependent i.e., they capture the group mobility behavior of the nodes, such as Exponential Correlated Random Mobility (ECRM) model [5], Pursue mobility model [18, 19] Column mobility model[18, 19], Reference point group mobility model [5] Reference Velocity Group Mobility model [20] and others. In Exponential correlated random mobility model, movement of the mobile nodes is directed by complex motion function, whereas the nodes in the Pursue mobility model, moves with little randomness towards the particular target. Nodes in the Column mobility model move in a forward direction around the line (or column). One of the most popular group mobility model is the Reference point group mobility model. In this, each node in the group is randomly distributed around the predefined reference point and follows the logical center of the group which decides the groups motion behavior along with a speed and direction via group motion vector $\overrightarrow{\mathrm{GM}}$. Reference Velocity Group Mobility model is another stochastic mobility model that uses the group velocity vector at time t to represent the group motion behavior.

Apart from individual and group mobility models, there are various other kinds of mobility models such as Obstacle mobility models like Pathway, Manhattan, Freeway mobility model, etc., which can model real world scenarios by avoiding environmental obstacles [21]. Map based mobility models are based on the traffic and user mobility patterns in order to evaluate and deal with networking issues with the wireless networks. A disaster aware mobility model is designed for flying Ad hoc Networks which is is emulated using map oriented navigation of nodes with realistic disaster situation [22]. In Social network based mobility model follows social network theories and very effectively captures the behavior of movement based on human decisions and socialization behavior like disaster relief, battlefield, etc. In [23] author proposed an N-body mobility model in which traces of a small number of nodes were captured for forming group behavior in order to reproduce them in the large population mobility traces. Inherently, social network theory studies the mobility pattern of the nodes based on social behavior for analyzing and formulating the network protocols in ad hoc networks.

However, our proposed AMIGM model is capable of modeling the migration of nodes from one group to another by replacing the worst fitness nodes and avoids the geographical restrictions, inter and intra group collision which was not present in the above existing mobility models. Moreover, AMIGM, RWP and RPGM models are evaluated with the protocol dependent and independent performance metrics under the DSR routing protocol.

3. Animal Migration Inspired Group Mobility Model. This Section discusses the proposed Animal Migration Inspired Group Mobility (AMIGM) model which is based on Animal Migration Optimization (AMO) algorithm [6] and some swarming laws for the governing of the group mobility.

The AMIGM model has two phases (1) Migration phase and (2) Population updating phase and it begins with the initialization process in which nodes are initially randomly distributed throughout the simulation area, according to a uniform distribution. In the migration phase, nodes within the group should follow three basic swarming rules: (1) they should remain close to their neighbors, (2) the whole group should move in the same direction to a common destination and (3) they should avoid inter and intra group collision. For the first and second rules, nodes should be kept within the sensor range $\rho$ ) of each other by calculating the distance of the nodes from each other through the Euclidean distance given below:

$$
d_{\text {min }}=\sqrt{\left(x_{1}-y_{1}\right)^{2}+\left(x_{2}-y_{2}\right)^{2}} \leq \rho
$$

Each group of nodes, then decides to move with the constant speed to a common destination or signal 
source which is randomly distributed throughout the simulation area with the desired homing heading angle $\psi_{H i}$, given in equation (3), mathematically proposed by Sharma and Ghose [24]].

The nodes in the network will be in the communication range of each other and will remain in one group $(\mathrm{G})$ if the distance $d_{\min }$ between the nodes is less than or equal to the sensor range $\rho$. After the formation of the group, neighbor list is defined for each node within the group $(G)$. Once the construction of the neighborhood topology gets completed, each node within the group moves from one position to another according to the neighbors position within the simulation area following the basic swarming laws. The $i^{\text {th }}$ node position of the group after $t+\Delta t$ time is updated according to the equation (2) and the updated position of the $i^{t h}$ node will be:

$$
X_{(i, t+\Delta t)}=X_{(i, t)}-\delta .\left(X_{(\text {neighborhood }, t)}-X_{(i, t)}\right) \leq \rho
$$

where, $X_{(\text {neighborhood,t })}$ is the current position of the neighborhood node and $\delta$ is the random number generator using Gaussian distribution.

Each group of nodes, then decides to move with the constant speed to a common destination or signal source which is randomly distributed throughout the simulation area with the desired homing heading angle $\psi_{H i}$, given in equation (3), mathematically proposed by Sharma and Ghose [24].

$$
\psi_{H i}=\arctan \left(\left(Y_{S i}-Y_{i}\right) /\left(X_{S i}-X_{i}\right)\right)
$$

where, $\left(X_{S i}, Y_{S i}\right)$ are the coordinates of the signal source.

For the third rule, nodes within the group avoids the collision with each other by maintaining the minimum required distance $d_{m i n}$ between the nodes with the desired dispersion heading angle $\psi_{D i}$, given in equation (4) $[24]$.

$$
\psi_{D i}=\arctan \left(\left(Y_{i}-Y_{C i}\right) /\left(X_{C i}-X_{i}\right)\right)
$$

where, $\left(X_{C i}, Y_{C i}\right)$ are the centroid of all the nodes within the group.

To avoid the collision between the groups they have to maintain the safe distance with the other group along with the minimization of group deviation from its current direction. The group first determines whether the other group is within the collision avoidance range $\rho_{c o l}$ or not. To maintain the brevity of the paper detailed explanation about this rule is not mentioned here, but can be found in [24]. For the collision avoidance rule, avoidance rule weight $W_{A V i}$ is given by:

$$
W_{A V i}= \begin{cases}1 & \text { if } P_{i}-C_{\rho, i} \leq \rho_{c o l} \\ 0 & \text { if } P_{i}-C_{\rho, i}>\rho_{c o l}\end{cases}
$$

The avoidance rule weight $W_{A V i}$ is used to calculate the desired heading angle $\psi_{A V i}$ :

$$
\psi_{A V i}=f_{1}\left(\frac{n}{2}\right)+\psi_{i}
$$

where, $\psi_{i}$ heading angle of the $i^{\text {th }}$ node and $f_{1}$ is the direction of the turn in the horizontal plane and is given by:

$$
f_{1}= \begin{cases}-1 & \text { if } \frac{\pi}{4}<\psi_{i} \leq \frac{3 \pi}{4} \text { and if } x_{i} \geq X_{c a} \\ +1 & \text { if } \frac{\pi}{4} \leq \psi_{i} \leq \frac{3 \pi}{4} \text { and if } x_{i}<X_{c a} \\ +1 & \text { if } \frac{5 \pi}{4}<\psi_{i} \leq \frac{7 \pi}{4} \text { and if } x_{i} \geq X_{c a} \\ -1 & \text { if } \frac{5 \pi}{4}<\psi_{i}<\frac{7 \pi}{4} \text { and if } x_{i}<X_{c a} \\ -1 & \text { if } \frac{3 \pi}{4}<\psi_{i} \leq \frac{5 \pi}{4} \text { and if } y_{i} \geq Y_{c a} \\ +1 & \text { if } \frac{3 \pi}{4} \leq \psi_{i}<\frac{5 \pi}{4} \text { and if } y_{i}<Y_{c a} \\ +1 & \text { if } \frac{7 \pi}{4}<\psi_{i} \leq \frac{\pi}{4} \text { and if } y_{i} \geq Y_{c a} \\ -1 & \text { if } \frac{3 \pi}{4} \leq \psi_{i}<\frac{5 \pi}{4} \text { and if } y_{i}<Y_{c a}\end{cases}
$$


In addition to the basic swarming rules, there is requirement of avoiding environmental obstacles for modeling the real world scenarios. In the proposed mobility model, the nodes avoid the collision with the environmental obstacles. The obstacles are fixed in our model and the nodes will avoid the obstacles if it detects an obstacle within its sensor range $\rho$; same as they avoid group collision mentioned in [24]. The desired obstacle avoidance rule heading angle $\psi_{O B i}$ is determined by [9]:

$$
\psi_{O B i}=w_{o b}\left(\frac{n}{4}\right)+\psi_{i}
$$

where, $w_{o b}$ is obstacle avoidance rule weight.

Superposition is used to calculate the desired heading angle $\psi_{r e q}$ given below as there are more than one swarming requirement rules in the proposed AMIGM model.

$$
\Delta \psi_{r e q}=w_{1}\left(\psi_{r e q 1}-\psi\right)+w_{2}\left(\psi_{r e q 2}-\psi\right)+\ldots+w_{n}\left(\psi_{r e q n}-\psi\right)
$$

The rule weights $w_{1}, w_{2}, ., w_{n}$ are constants and varied to obtain different basic behaviors, where $\left|w_{i}\right| \leq 1$ and $\psi$ is the heading angle of the node.

In the population updating phase, some nodes will leave the group and some nodes will migrate from one group to another group. The nodes with best fitness value will replace the nodes with worst fitness value during the migration of the nodes. In the AMIGM model, each node of the group randomly moves within the simulation area and if the groups of node is within the specified range $\rho_{G}$, the nodes will migrate to another group and replace the node with worst fitness value with the probability $p_{a}$.

$$
D_{\min }=\sqrt{\left(C_{G x i}-C_{G y_{i}}\right)^{2}} \leq \rho_{G}
$$

where, $\left(C_{G x i}, C_{G y_{i}}\right)$ is the centroid of the group of nodes and $\rho_{G}$ is the sensor range of the group of nodes.

The residual energy of the node is used as the fitness function for evaluating the solution. The AMIGM model makes use of nodes energy as the fitness function for evaluating the solution. Suppose $\mathrm{N}$ nodes $N_{1}, N_{2},, N_{n}$ of group $G_{i}$ are randomly distributed in the simulation area and each node of the group $G_{i}$ has initial energy, $E_{0}$. The random $m$ destination or target $d_{1}, d_{2},, d_{m}$ is defined for each group of nodes $G_{i}$ for visiting within the simulation environment. The visiting matrix $V_{i, j}$ of the group of nodes $G_{i}$ is given in equation (11). If the nodes in the group $G_{i}$ reach or visit the randomly selected destination $d_{j}$ the visiting matrix $V_{i, j}$ will be:

$$
V_{i, j}= \begin{cases}1 & \text { if } N_{i} \text { ofeach } G_{i} \text { visitsthetarget }_{j} \\ 0 & \text { otherwise }\end{cases}
$$

where $i=1,2, n$ and $j=1,2, m$

If $b_{i}$ is the initial power of the battery and $e_{i}$ is the energy consumption rate then the energy of the node will be:

$$
b_{i}^{\prime}=\frac{b_{i}}{e_{i}}
$$

Hence, for the evaluation of the solution the energy of the node will be the fitness function which is given by:

$$
f_{x}=\left(V_{i, j} * b_{i}{ }^{\prime}\right)
$$

The probability of a node being selected in the BIGM model with $f_{x}$ as the fitness of a node is:

$$
P_{a}=\frac{f_{x}}{\sum_{i=1}^{n} f_{y}}
$$

For the worst fitness, the probability $P_{a}$ is $1 / \mathrm{N}$ where, $\mathrm{N}$ is the number of nodes in the network and for the best fitness the $N$ probability $P_{a}$ is 1 . 


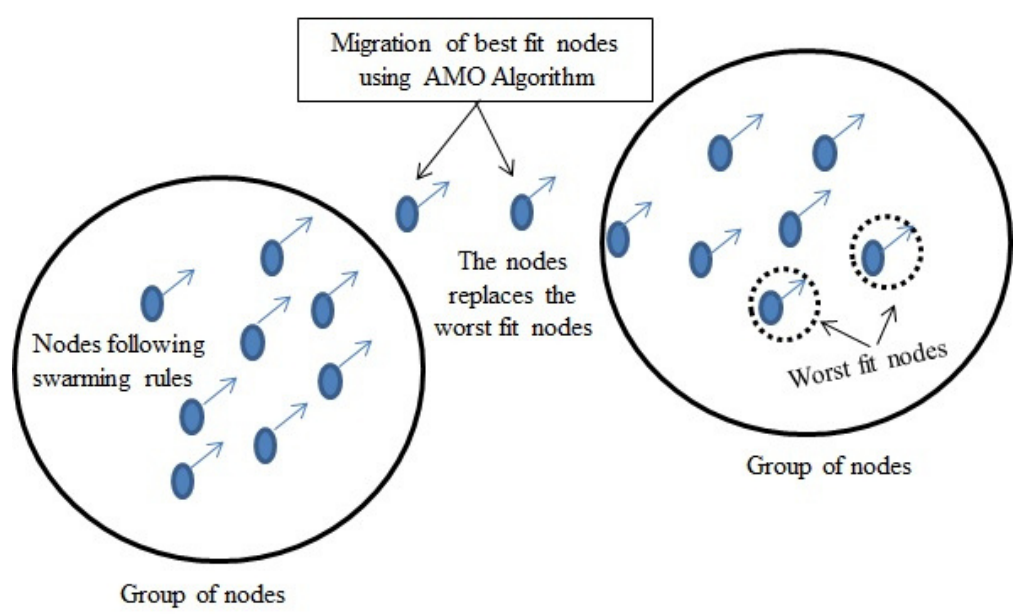

FIG. 3.1. Migration of the nodes using Animal Migration Optimization Algorithm.

The proposed AMIGM algorithm will select the best fitness value nodes i.e., the nodes with maximum amount of energy to migrate to another group of nodes. If the probability $P_{a}$ of a node is greater than that of a randomly generated number (i.e., between 0.0 and 1.0) then that node will be selected for the migration with best fitness value. The selected node is then compared with the worst fitness node from the other group, and if the selected node has better fitness value, then the selected node will migrate into that group replacing the worst fitness node otherwise selected node will not migrate. After migrating to other group the selected node move towards the target or destination $d_{j}$, along with that group.

AMIGM model is designed for modelling the movement pattern of the nodes in wireless ad hoc networks and could be applied to a diverse range of the mobile ad hoc application scenarios. If we take a scenario of military application, where there are different teams like a disaster management team, rescue team, medical assistant team etc., working on the same battlefield, then there are chances that the medical assistant team may give some assistance to the disaster recovery team depending upon the application scenario. Then, some or whole medical assistant team may join the rescue team over the same disaster recovery area and work with them as a whole by replacing the worst node (in terms of energy of the nodes) of the rescue team by the best node of the medical assistant team. As there are the chances that either of the teams may have nodes with less energy while moving within the application scenario, then the nodes with the best energy will replace the worst energy nodes from either of the teams as required. It can be seen that in these types of application scenarios AMIGM model can work well.

4. Animal Migration Inspired Group Mobility Model Algorithm. The Animal Migration Inspired Group Mobility Model (AMIGM) is discussed above in Section 3 and the algorithm for the above proposed model is as follows:

Algorithm 1 Animal Migration Inspired Group Mobility Model for Mobile Ad hoc Networks

Input: Number of nodes N, Number of group of nodes G and Sensor range $\rho$.

Output: Migration of nodes to another group by replacing the worst fit nodes.

1: begin

2: $\quad$ Create a network of nodes $\mathrm{N}$ and set initial position of nodes according to a uniform distribution.

3: $\quad$ Define a neighbor list for each node.

4: $\quad$ Define group $\mathrm{G}$ for the network by calculating distance and comparing it with sensor range $\rho$ of each node $\mathrm{N}$ in the network.

5: $\quad$ Randomly select a destination for the group.

6: $\quad$ Allot energy to each node $\mathrm{N}$ in the group.

7: $\quad$ while maximum simulation time do 


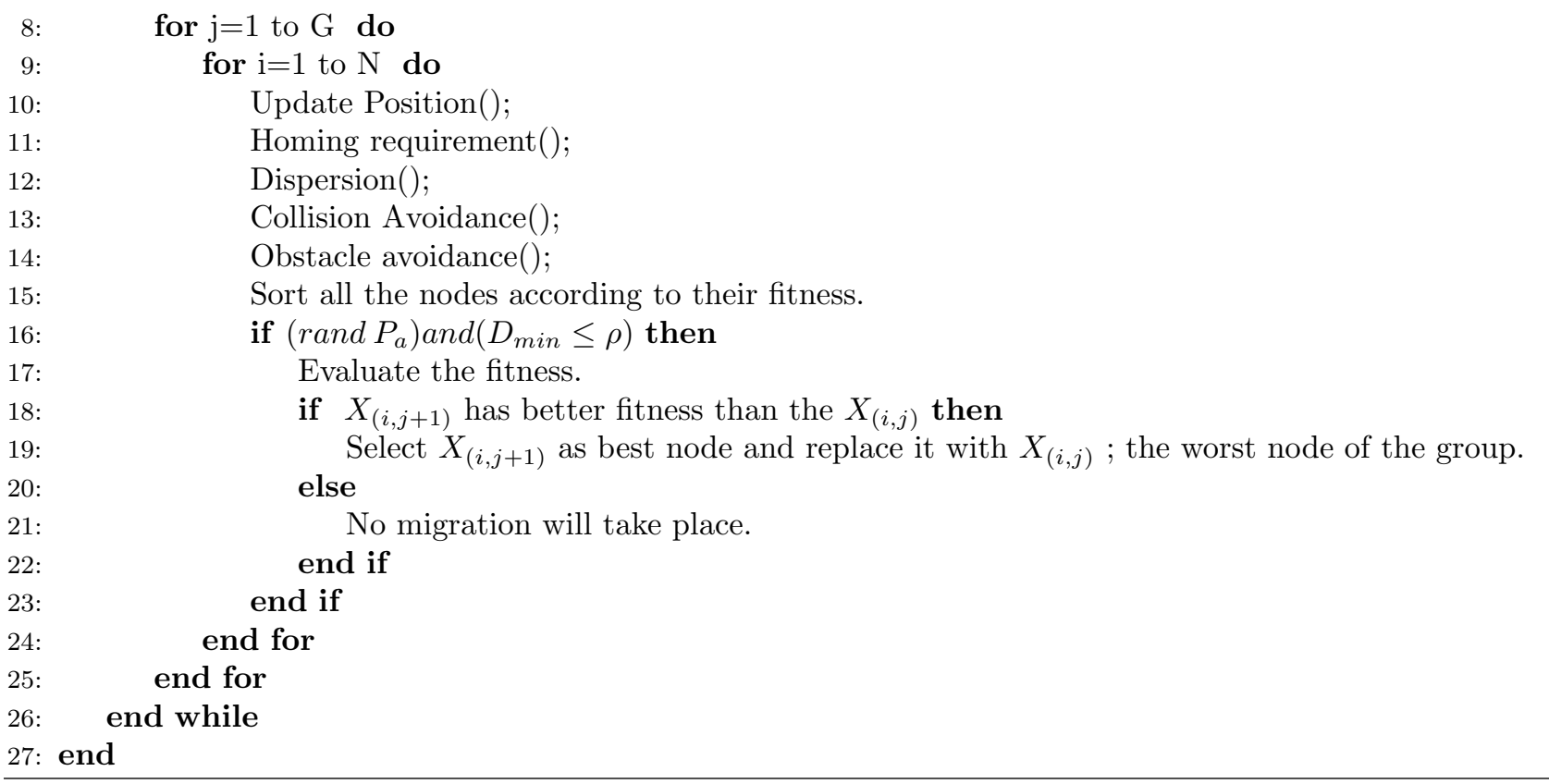

5. Complexity Analysis. This section analyses the time complexity of the proposed AMIGM algorithm. In the proposed algorithm for AMIGM model (see algorithm 1), neighbors of the node will get updated each time it iterates through n nodes; hence, the complexity will be $O\left(n^{2}\right)$ for the updated list of neighborhood. The selection of the best fit nodes from the group $\left(G_{i}\right)$ and comparing that best fit node with the worst fit node, the algorithm requires the complexity of $O\left(n^{2}\right)$ in worst case and to replace the worst fit node with the best fit selected nodes constant time will be required during migration of nodes in the algorithm. Hence, for each group $\left(G_{i}\right)$ the migration algorithm iterates through $\mathrm{n}$ nodes and has the complexity of $O\left(n^{2}\right)$. Therefore, the complexity of the proposed AMIGM algorithm will be $O\left(n^{2}\right)$.

6. Simulations and Results. This Section presents the simulation environment and configurations used for testing the AMIGM model. Also, a comparative result of the AMIGM model with the RWP and RPGM model is evaluated under the DSR routing protocol.

6.1. Simulation Environment. The simulator used for modeling the proposed mobility model was ns-2 [25] and cbrgen tool of ns-2 was used for generating the communication traffic. We have randomly chooses the source and destination pairs with 30 independent cbr traffic sources and overall 30 connections. Each simulation result of the mobility models used setdest tool to generate mobility scenarios and has eight different mobility scenario files for the maximum speed of $5,10,15,20,25,30,35$ and $40 \mathrm{~m} / \mathrm{s}$ with a 0 s pause time. Also, we have considered 10 randomly distributed obstacles within the simulation area in our proposed model. The performance metrics of the proposed model along with the RWP and RPGM model under the DSR routing protocol are compared. The nominal values of the weights discussed in Section 3 are assumed to be $w_{c}=0.5$, $w_{h}=0.7, w_{d}=0.5$ and $w_{a v}=0$, without collision avoidance scheme. With collision avoidance scheme values will be $w_{c}=0.25, w_{h}=0.35, w_{d}=0.5$ and $w_{a} v=1$. The values of the weights are borrowed from Sharma and Ghose [24]. For the obstacle avoidance scheme if an obstacle is detected within the sensor range of the nodes the value of $w_{o b}$ will be 1 and if no obstacle is detected then the value of $w_{o b}$ will be 0 . The parameters used for conducting the simulation of mobility models are given in Table 6.1 and the distribution of the nodes in the mobility models are given in Table 6.2.

6.1.1. Average Degree of Spatial Dependence. It is the measure of similarities of node velocities of the two neighboring nodes. In AMIGM model, nodes are spatially correlated as the group of nodes try to keep up common heading angle. As can be seen in Fig. 6.1 AMIGM mobility model has the highest average degree of spatial dependence compared to RPGM and RWP as in the AMIGM model all the nodes belonging to a group move towards the same direction with the same speed. For instance, AMIGM shows the highest average degree 
Simulation Parameters.

\begin{tabular}{|l|l|}
\hline Simulation Parameters & \multicolumn{1}{|c|}{ Specifications } \\
\hline Simulation Time & $800 \mathrm{~s}$ \\
Field Dimensions & $(1500 \mathrm{~m}, 1500 \mathrm{~m})$ \\
Wireless Antenna & Omni directional antenna \\
Network Interface & Wireless \\
Channel & Wireless \\
Initial Energy & 60 Joules \\
Transmission Range & $250 \mathrm{~m}$ \\
Interface Queue & CMU Priority queue, Queue/DropTail/PriQueue \\
Interface queue length & 50 \\
Propagation path loss & Two ray ground \\
MAC Protocol & 802.11 \\
Routing Protocol & DSR, AODV, DSDV \\
Data Rate & $200 \mathrm{~kb}$ \\
CBR Packet Size & 64 bytes \\
UDP Packet Size & 512 \\
\hline
\end{tabular}

TABLE 6.2

Node Distribution in Groups with Different Node Density.

\begin{tabular}{|c|c|c|}
\hline Mobility Models & Groups & Nodes/Group \\
\hline RWP & No Groups & 60 \\
RPGM & 1 & 35 \\
& 2 & 25 \\
AMIGM & 1 & 35 \\
& 2 & 25 \\
\hline
\end{tabular}

of spatial dependence of 0.51 at the maximum speed of $25 \mathrm{~m} / \mathrm{s}$ as compared to RPGM and RWP which shows 0.28 and 0.00052 average degree of spatial dependence respectively.

6.1.2. Average Link Duration. Link duration is the measure of the link duration between the nodes in the network. Nodes in the ad hoc wireless networks frequently change the topology and hence, the nodes exhibit more links up/down with high mobility. But the nodes in the AMIGM mobility model remain close to each other because of the cohesive property explained in Section 3 and thus, AMIGM mobility model shows the highest average link duration because of the fewer links up/down between the nodes compared to RPGM and RWP mobility models (see Fig. 6.2). It is desirable that the average link duration remains high for the energy constraint MANETs. For instance, the average link duration of the AMIGM mobility model is $17.2 \mathrm{~s}$ at the maximum speed of $25 \mathrm{~m} / \mathrm{s}$ as compared to RPGM and RWP mobility model which shows $10.34 \mathrm{~s}$ and 3.37 s respectively. As shown in Fig. 6.2 with high mobility the average link duration between the nodes decreases with an increase in maximum speed as they exhibit more links up/down.

6.1.3. Packet Delivery Ratio. The packet delivery ratio is defined as the ratio of the successfully received packets by the destination to the generated packets by the source. In Fig. 6.3 we can see that RPGM mobility model has the highest packet delivery ratio as compared to RWP and AMIGM models for DSR routing protocol as nodes in AMIGM model avoids the inter-group communication as a result of which most of the routing packets are dropped. But in some instances, AMIGM shows the highest packet delivery ratio than RPGM. 


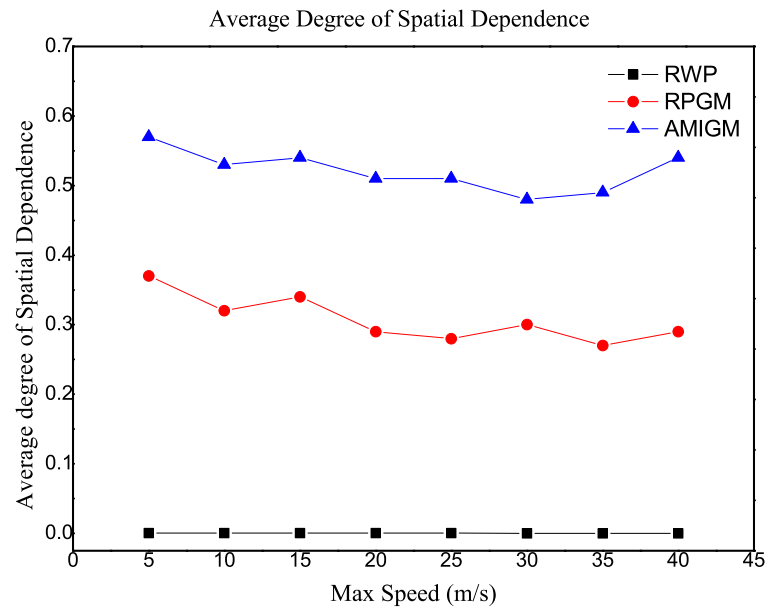

FIG. 6.1. Average Degree of Spatial Dependence with the varying network load across various mobility models.

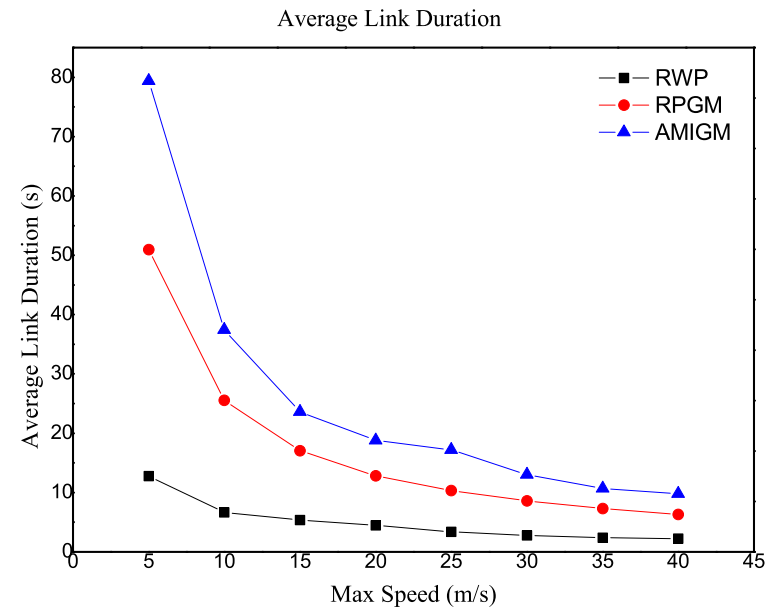

FIG. 6.2. Average Link Duration with the varying network load across various mobility models.

For instance, in Fig. 6.3 for the DSR routing protocol when the maximum speed is $5 \mathrm{~m} / \mathrm{s}$, AMIGM shows the highest packet delivery ratio of 1.11 compared to RWP and RPGM which shows 0.34 and 1.21 respectively. Thus, from the Fig. 6.3 it is clear that in some instances, AMIGM shows the highest packet delivery ratio than RWP and RPGM mobility as in those instances, due to the migration of nodes from one group to another group, the nodes remain within the transmission range of each other.

6.1.4. Normalized Routing Overhead . Normalized routing overhead is defined as the total number of routing packets sent per data packet to the destination. In Fig. 6.4 we can see that RWP mobility model has the highest normalized routing overhead as nodes move independently in RWP and thus route fluctuate more rapidly compared to RPGM and AMIGM models for the DSR routing protocol. For instance, in Fig. 6.4 for DSR routing protocol, when the maximum speed is $25 \mathrm{~m} / \mathrm{s}$ the AMIGM shows the routing overhead of 0.75 kbps whereas RWP and RPGM show the routing overhead of $1.55 \mathrm{kbps}$ and $0.59 \mathrm{kbps}$ respectively. Thus, from 


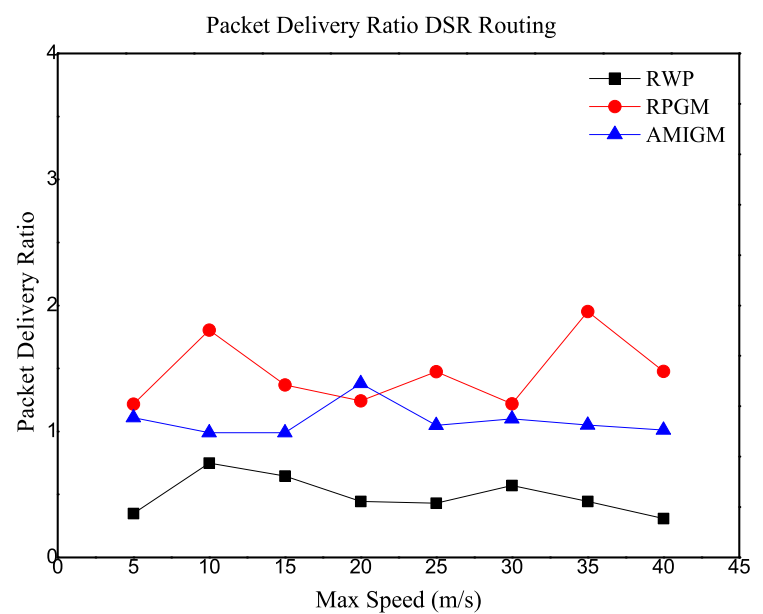

FIG. 6.3. Packet Delivery Ratio (in fraction) across various mobility models with maximum speed for DSR with AMIGM.

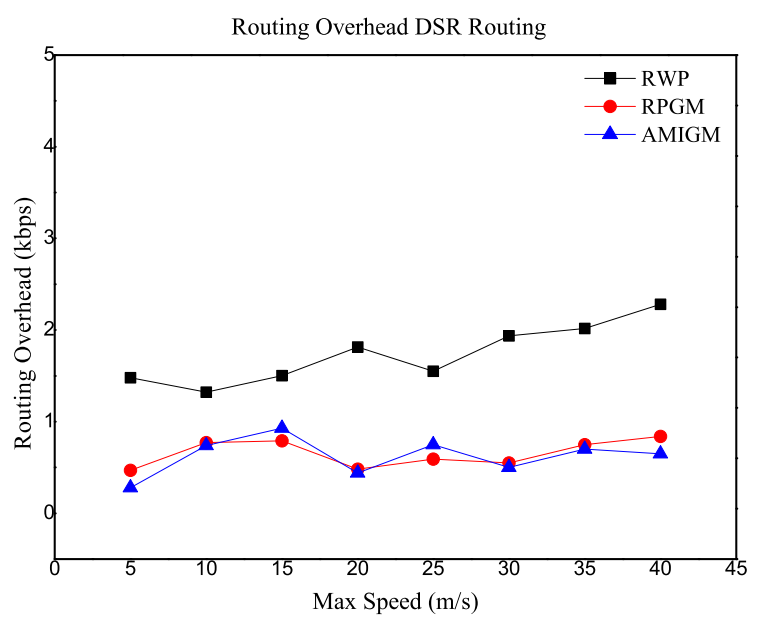

FIG. 6.4. Normalized Routing Overhead (control packets per data packets sent) across various mobility models with maximum speed for DSR with AMIGM.

the Fig. 6.4, it is clear that after RWP, AMIGM shows the highest normalized routing overhead as compared to RPGM model.

6.1.5. End-to-End Delay . End-to-End Delay is defined as the amount of time, a bit of data will take to travel between the two communicating nodes. In Fig. 6.5 we have observed that the RWP mobility model shows the maximum delay as compared to RPGM and AMIGM models for the DSR routing protocol. For instance, in Fig. 6 at the maximum speed of $40 \mathrm{~m} / \mathrm{s}$ RWP, RPGM and AMIGM shows the delay of $0.80 \mathrm{~s}, 2.51 \mathrm{~s}$, and 2.72 s respectively. Thus, from the Fig. 6.5, it is concluded that AMIGM shows the maximum delay compared to the RPGM model because with the inter-group communication the connection setup delay is high.

6.1.6. Throughput. Throughput is defined as the amount of data successfully sent from one node to another in a specified period of time. In Fig. 6.6, we have observed that the RPGM mobility model has the 


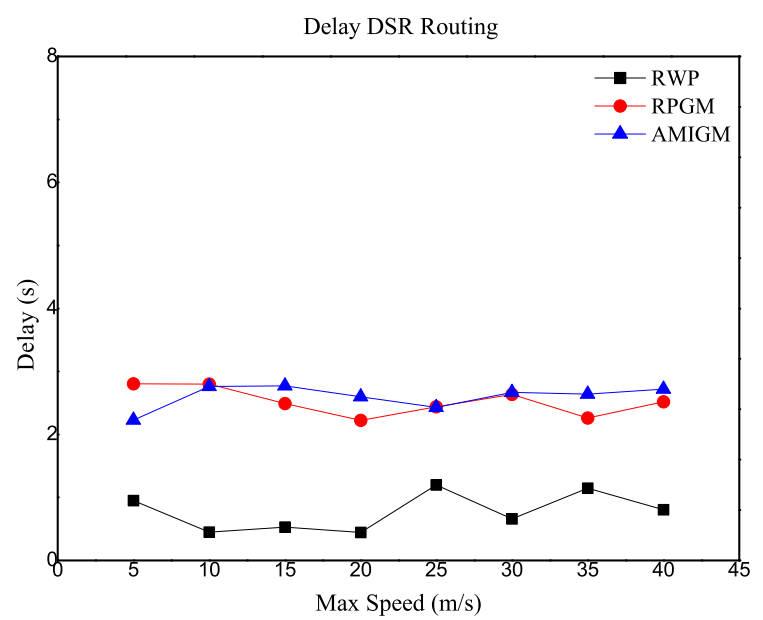

FIG. 6.5. End-to-End Delay (in fraction) across various mobility models with maximum speed for DSR with AMIGM.

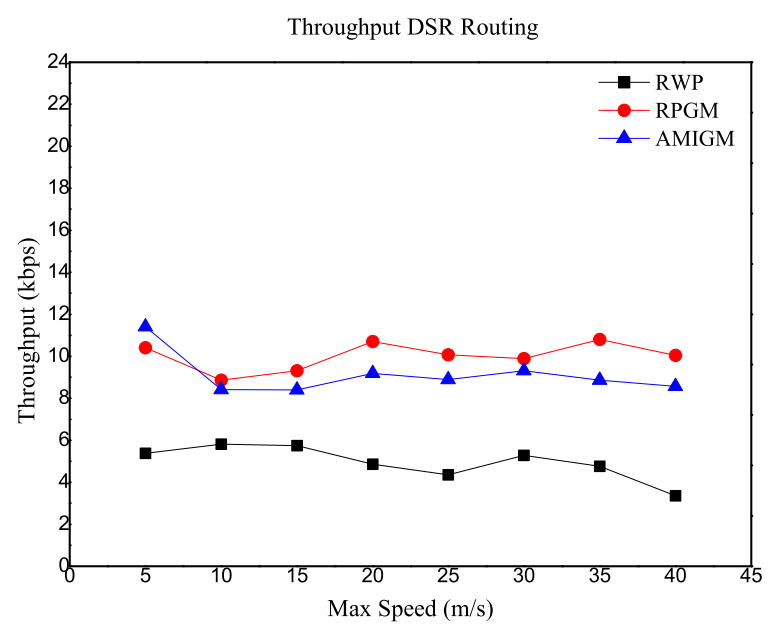

FIG. 6.6. Throughput (kbps) across various mobility models with maximum speed for DSR with AMIGM.

highest throughput as compared to RWP and AMIGM models for the DSR routing protocol because AMIGM model avoids the inter-group communication. In Fig. 6.6 with DSR routing protocol at the maximum speed of $5 \mathrm{~m} / \mathrm{s}$ AMIGM shows the throughput of $11.4 \mathrm{kbps}$ whereas RWP and RPGM models shows the throughput of $5.38 \mathrm{kbps}$ and $10.4 \mathrm{kbps}$ respectively. Thus, from the Fig. 6.6, it is concluded that in some instances, AMIGM shows the maximum throughput than RPGM model as the nodes in AMIGM model are within the transmission range of each other due to the migration of nodes from one group to other.

6.1.7. Average Energy Consumption. It is the amount of energy consumed during the movement of nodes in the network. In Fig. 6.7 we have observed that the RPGM mobility model has the highest energy consumption as compared to RWP and AMIGM models for the DSR routing protocol. In Fig. 6.7with DSR routing protocol at the maximum speed of $35 \mathrm{~m} / \mathrm{s}$ AMIGM shows the average energy consumption of 57.62 joules whereas RWP and RPGM models show the average energy consumption of 59.00 joules and 58.12 joules 


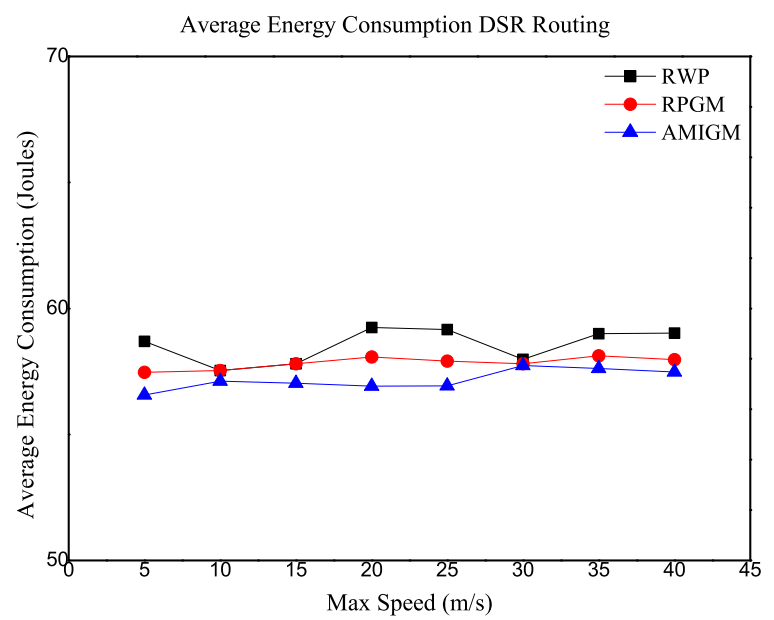

FIG. 6.7. Average energy consumption (joules) across various mobility models with maximum speed for DSR with AMIGM.

respectively. Thus, from the Fig. 6.7, it is concluded that AMIGM shows the minimum average energy consumption than RPGM model as the nodes in AMIGM model has the highest average spatial dependences and link duration. In addition, it also avoids the obstacle collision, inter and intra group collision between the nodes because of that least energy is consumed by the AMIGM model.

7. Movement Pattern of Nodes in Mobility Models. The mobile nodes in wireless ad hoc networks exhibit different movement pattern for modeling the realistic scenarios. Mobility models with their different parameters exhibit different characteristics which in turn affect the network protocols and can be used to evaluate network protocols for different application scenarios.

Following are some mobility model's characteristics for modelling the realistic scenarios:

1. Temporal dependencies: Mobility behavior in realistic scenarios, such as sudden change in acceleration, sharp turns and sudden stop may not occur frequently and the real movement of nodes defines the temporal dependencies based on the past movement of nodes. Random based mobility model are not able to model the real world scenarios as the velocity in these models has memoryless behavior.

2. Spatial dependencies: In most real world scenarios the nodes hardly move independently rather they move in a correlated fashion, such as military operations, emergency operations, conferences, museum touring and security operations. The mobility of the nodes depends on the mobility behavior of neighboring nodes which is absent in random based mobility models because the nodes move independently.

3. Geographical dependencies: Real world scenarios have many environmental restrictions like, buildings, trees, vehicles etc., and so, mobility models must take geographical restrictions into consideration while designing the models. Random mobility models do not take geographical restriction into consideration as they move freely within the simulation area and fail to model the real world scenarios.

The comparisons of different mobility models with the proposed AMIGM model under temporal, spatial dependencies and geographical restrictions are presented in Table 7.1.

As discussed above, random based mobility models are not spatial and temporal dependent, but nodes in synthetic mobility models like RPGM model move in a group and are correlated with each other without imposing geographical restrictions, hence RPGM model is spatial dependent. The proposed AMIGM model is temporal as well as the spatial dependent because nodes in AMIGM model move in a group as a whole by using basic swarming laws and movement of nodes do not include sharp turns rather they move incrementally while avoiding environmental obstacles present in the real world scenarios. 
TABLE 7.1

Comparison of Mobility Models

\begin{tabular}{|c|c|c|c|}
\hline Mobility Models & Temporal Dependency of Velocities & Spacial Dependency of Velocities & Geographical Restrictions \\
\hline RWP & No & No & No \\
RPGM & No & Yes & No \\
AMIGM & Yes & Yes & Yes \\
\hline
\end{tabular}

8. Conclusion. A realistic mobility model is very significant for the evaluation of the performance of network protocols and validation of real world traces in the wireless ad hoc networks. This paper proposes an Animal Migration Inspired Group Mobility Model (AMIGM) which tries to mimic the movement of the real mobile node during migration from one group to another using AMO algorithm. The proposed model follows the simple swarming rules for the movement and the formation of the group of nodes. Also, it is capable of avoiding inter-intra group collision, environmental obstacles and can stimulate the migration of nodes between the groups by replacing the worst fitness nodes. We have simulated and compared the performance of the proposed AMIGM model with the RWP and RPGM mobility models under the DSR routing protocol and connectivity metrics. The simulation results for the proposed AMIGM model shows that the model has highest average link duration and average degree of spatial dependence with least average energy consumption when compared with other existing mobility models (RWP and RPGM) under the DSR routing protocol.

\section{REFERENCES}

[1] C. S. R. Murthy, Ad hoc wireless networks: Architectures and protocols, Pearson Education, India, 2004.

[2] T. CAmp, J. Boleng, V. Davies, A survey of mobility models for ad hoc network research, Wireless communications and mobile computing,2(5) (2002), pp. 483-502.

[3] M. L. Sichitiu, Mobility Models for Ad hoc Networks, in Guide to Wireless Ad Hoc Networks, Springer, London, 2009, pp. $237-147$.

[4] D. B. Johnson, And D. A. Maltz, Dynamic Source Routing in Ad hoc Wireless Networks, in Mobile Computing, Springer, Boston, MA, 1996.

[5] X. Hong, M. Gerla, G. Pei, And C. C. Chinng, A Group Mobility Model for Ad hoc Wireless Networks, in Proceedings of the 2nd ACM International Workshop on Modeling, Analysis and Simulation of Wireless and mobile Systems, ACM, August 1999, pp. 53-60.

[6] X. Li, J. Zhang, AND M. YIN, Animal migration optimization: an optimization algorithm inspired by animal migration behavior, Neural Computing and Applications, 24(7-8) (2014), pp.1867-1877.

[7] J. Verma, And N.Kesseani, A Review on Bio-Inspired Migration Optimization Techniques, International Journal of Business Data Communications and Networking (IJBDCN), 11 (2015), pp. 24-35.

[8] B. Zhou, K. Xu, And M. Gerla, Group and Swarm Mobility Models for Ad hoc Network Scenarios using Virtual Tracks in Military Communications Conference, MILCOM 2004, IEEE, 1 (October 2004), pp. 289-294.

[9] S. Misra, And P. Agarwal, Bio-Inspired Group Mobility Model for Mobile Ad hoc Networks based on Bird-Flocking Behavior, Soft Computing, 16(3) (2012), pp. 437-450.

[10] J. Huo, B. Deng, S. Wu, J. Yuan, And I. You, A topographic-Awareness and Situational-Perception based Mobility Model with Artificial Bee Colony Algorithm for Tactical MANET, Computer Science and Information Systems, 10(2) (2013), pp. $725-746$.

[11] J. Verma, And N. Kesswani, BIGM: A Biogeography Inspired Group Mobility Model for Mobile Ad Hoc Networks, International Journal of Wireless Information Networks, 25(4) (2018), pp. 488-505.

[12] F. Bai, And A. Helmy, A Survey of Mobility Models, in Wireless Adhoc Networks, University of Southern California, USA, 206,147

[13] J. M. NG AND Y. ZHANG, Reference region group mobility model for ad hoc networks, in Proceedings of Second IFIP International Conference on Wireless and Optical Communications Networks, IEEE, 2005, pp. 290-294.

[14] A. Einstein, Investigations on the Theory of the Brownian Movement, Courier Corporation, 1956.

[15] E. M. Royer, P. M. Melliar-Smith, and L. E. Moser, An Analysis of the Optimum Node Density for Ad hoc Mobile Networks, in Communications, 2001, ICC 2001, IEEE International Conference on, 3 (2001), pp. 857-861.

[16] Z. Gong, AND M. HAENGGI, Interference and outage in mobile random networks: Expectation, distribution, and correlation, IEEE Transactions on Mobile Computing, 13(2) 2014, pp. 337-349.

[17] Y. CONG, X. Zhou, AND R. A. Kennedy, Interference prediction in mobile ad hoc networks with a general mobility model,IEEE Transactions on Wireless Communications, 14(8) 2015, pp. 4277-4290.

[18] M. Bergamo, R. R. Hain, K. Kasera, D. Li, R. Rananathan, and M. Steenstrup, System Design Specification for Mobile Multimedia Wireless Network $(M M W N)($ draft), DARPA project DAAB07-95-C-D156, 1996.

[19] SAnchez, Mobility Models, http://www.disca.upv.es/misan/mobmodel.htm, page accessed on October 15 th 2017. 
[20] K. H. Wang, and B. Li, Group mobility and partition prediction in wireless ad-hoc networks, in Proceedings of Communications, ICC 2002, IEEE International Conference on. 2, IEEE, 2002, pp. 1017-1021.

[21] F. Bai, N. Sadagopan, and A. Helmy, ImPORTANT: A Framework to Systematically Analyze the Impact of Mobility on Performance of Routing Protocols for Adhoc NeTworks, in INFOCOM 2003, Twenty-Second Annual Joint Conference of the IEEE Computer and Communications, IEEE Societies, IEEE, 2 (March 2003), pp. 825-835.

[22] A. Mukherjee, N. Kausar, A. S. Ashour, R. Taiar, and A. E. Hassanien, A disaster management specific mobility model for flying ad-hoc network, Emergency and Disaster Management: Concepts, Methodologies, Tools, and Applications, 25, 2019, pp. 279-311.

[23] C. Zhao, M. L. Sichitiu, And I. Rhee, N-body: A social mobility model with support for larger populations, Ad Hoc Networks, 25, 2015, pp. 185-196.

[24] R. K. Sharma, And D. Ghose, Collision Avoidance between UAV Clusters using Swarm Intelligence Techniques, International Journal of Systems Science, 40(5) (2009), pp. 521-538.

[25] The VINT Project, The network simulator ns-2, http://www.isi.edu/nsnam/ns/, page accessed on 6 Jan 2016.

\section{Edited by: Dana Petcu}

Received: June 18, 2019

Accepted: September 9, 2019 